\title{
CCR3- and CXCR4-mediated interactions regulate migration of CD34 + human bone marrow progenitors to ischemic myocardium and subsequent tissue repair
}

\author{
N. Bonaros, MD, ${ }^{a, *}$ H. Sondermejer, MD, ${ }^{b, *}$ M. Schuster, PhD, ${ }^{b}$ R. Rauf, MD, ${ }^{a}$ S. F. Wang, MD, ${ }^{b}$ T. Seki, PhD, \\ D. Skerrett, MD, ${ }^{b}$ S. Itescu, MD, ${ }^{b}$ and A. A. Kocher, MD ${ }^{a, b}$
}

From the Department of Cardiac Surgery Innsbruck Medical University, Innsburck, Austria, ${ }^{a}$ and the Departments of Medicine, Surgery, and Pathology, Columbia University, New York, NY. ${ }^{\mathrm{b}}$

Michael Schuster reports consulting fees and equity from Angioblast Systems and Mesoblast Limited; he is an employee of Angioblast Systems. Silviu Itescu reports consulting fees and equity from Angioblast Systems and Mesoblast Limited; he is an officer of Angioblast Systems. Angioblast Systems has a license for production of eotaxin.

* These authors contributed equally.

Received for publication Oct 5, 2007; revisions received Dec 6, 2007; accepted for publication Dec 24, 2007.

Address for reprints: Nikolaos Bonaros, MD, Department of Cardiac Surgery, Innsbruck Medical University, Anichstrasse 35, A-6020, Innsbruck, Austria (E-mail: nikolaos.bonaros@i-med.ac.at).

J Thorac Cardiovasc Surg 2008;136:1044 53

\section{$0022-5223 / \$ 34.00$}

Copyright $\odot 2008$ by The American Association for Thoracic Surgery

doi:10.1016/j.jtcvs.2007.12.067
Objective: Hematopoietic progenitor cells are able to induce neovascularization of ischemic myocardium, inhibit apoptosis, and prevent heart failure. They express functional CC chemokine-binding receptor 3 (CCR3) and CXC chemokine-binding receptor 4 (CXCR4); however, the role of those receptors in migration of progenitor cells into the ischemic myocardium is unknown.

Methods: Myocardial infarction was surgically induced in athymic nude rats, and human bone marrow-derived CD34+ cells or saline was injected into the tail vein. Cell chemotaxis was studied in vitro using chemotaxis chambers with or without concomitant stimulation with eotaxin or stromal cell-derived factor-1. Cell migration into ischemic myocardium was evaluated by immunohistochemistry. CCR3 and CXCR4 antibodies or local injections of stromal cell-derived factor-1 were used to investigate the role of chemokine expression in the migration capacity of the injected cells. Morphologic analysis included evaluation of apoptosis and capillary density in the ischemic myocardium.

Results: Ischemic rat myocardium demonstrated induced messenger RNA expression for the CCR3-binding chemokines eotaxin, RANTES (regulated on activation, normal $\mathrm{T}$ expressed and secreted), and monocyte chemotactic protein-3, but not the CXCR4-binding chemokine stromal cell-derived factor-1. Migration of human angioblasts to ischemic rat myocardium was inhibited by a blocking anti-CCR3 monoclonal antibody, but not by a blocking anti-CXCR4 monoclonal antibody, which instead inhibited migration to bone marrow. Finally, intramyocardial injection of stromal cell-derived factor-1 redirected migration of human angioblasts to ischemic rat hearts, resulting in augmented neovascularization, enhanced cardiomyocyte survival, and functional cardiac recovery.

Conclusions: CCR3-dependent chemokine interactions regulate endogenous migration of $\mathrm{CD} 34+$ progenitors from bone marrow to ischemic but not to normal myocardium. Manipulating CXCR4-dependent interactions could enhance the efficacy of cell therapy after myocardial infarction.

I $\mathrm{n}$ normal adult animals, CD34+ hematopoietic progenitors continuously migrate between the bone marrow and the intravascular compartment. ${ }^{1}$ Bone marrow homing and retention of $\mathrm{CD} 34+$ hematopoietic progenitors in adult mammals are processes controlled by a number of adhesive interactions, ${ }^{2,3}$ including those between the CXC chemokine stromal cell-derived factor-1 (SDF-1), which is constitutively produced by bone marrow stromal cells, and its receptor, CXCR $4 .{ }^{4,5}$ Disruption of bone marrow SDF-1/CXCR4 interactions, ${ }^{6}$ as occurs after activation of neutrophil proteases by systemic administration of granulocyte-colony stimulating factor (G$\mathrm{CSF}$ ), results in transient egress of hematopoietic progenitors from the bone marrow into the peripheral circulation, a phenomenon termed mobilization. ${ }^{7}$ These observations form the basis of clinical protocols enabling large scale harvesting of bone 


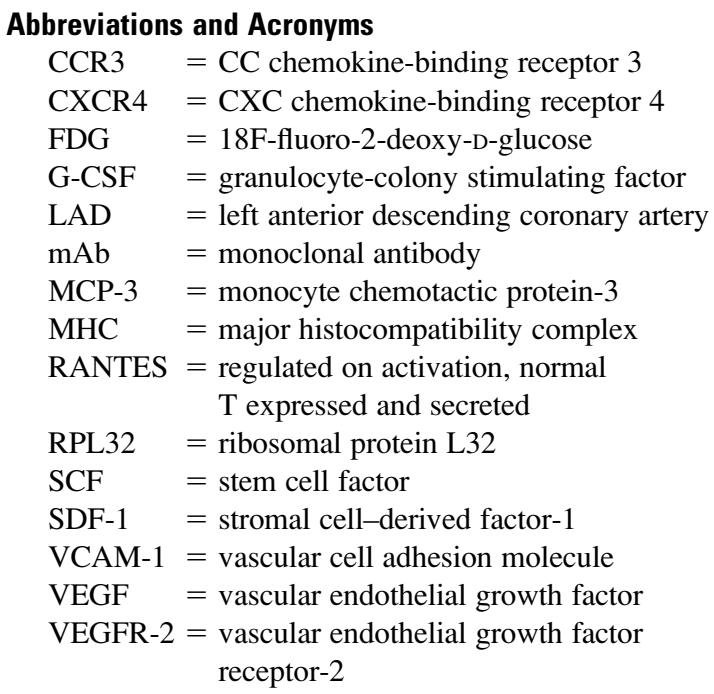

marrow CD34+ progenitors for autologous stem cell transplantation in certain hematologic disorders.

Bone marrow-derived CD34+ progenitors are capable of giving rise to cells of nonhematopoietic lineage, including hepatocytes, ${ }^{8}$ epithelial cells, ${ }^{9}$ and endothelial cells. ${ }^{10} \mathrm{We}^{10}$ have reported that G-CSF-mobilized human adult bone marrow elements contain $\mathrm{CD} 34+$ progenitors with phenotypic and functional characteristics of embryonic angioblasts that, when transplanted into animal models of acute myocardial infarction, home to ischemic myocardium, induce neovascularization, and result in improved cardiac outcome. Similar results have been obtained with autologous bone marrow cells in humans with acute myocardial ischemia. ${ }^{11,12}$ Collectively, these studies suggest that a general mechanism by which endogenous repair of damaged tissues occurs is redirected migration of $\mathrm{CD} 34+$ progenitors from the bone marrow to sites of acute injury, including the heart.

In adult mice, bone marrow progenitors capable of hematopoietic reconstitution have been reported to constitutively express several chemokine receptors, including CXC chemokine-binding receptor 4 (CXCR4) and CC chemokine-binding receptor 3 (CCR3), but to demonstrate restricted chemotactic responses only to SDF- $1 .{ }^{13}$ In contrast, human CD34+ bone marrow and cord blood cells, which contain both hematopoietic and nonhematopoietic progenitors, demonstrate robust chemotactic responses to diverse chemokines in addition to SDF-1, including migration to nonhematopoietic sites in response to the CCR3-binding CC chemokine eotaxin. ${ }^{14,15}$ In this study, we investigated the nature of the endogenous chemotactic signals provided by ischemic myocardium that result in migration of human bone marrow-derived angioblasts to the heart to identify strategies to enhance cell migration into the ischemic myocardium.

\section{Methods}

\section{Purification and Characterization of Cytokine-} mobilized Human CD34+ Cells

Single-donor leukopheresis products were obtained from humans treated with recombinant G-CSF $10 \mu \mathrm{g} / \mathrm{kg}$ (Amgen, Thousand Oaks, Calif) subcutaneously daily for 4 days after approval by the ethical committee of Columbia University. Mononuclear cells were separated by Ficoll-Hypaque, and highly purified CD34+ cells ( $>98 \%$ positive) were obtained using magnetic beads coated with anti-CD34 monoclonal antibody (mAb) (Miltenyi Biotech, Auburn, Calif). Purified CD34 cells were stained with fluoresceinconjugated mAbs against CD34 and CD117 (Becton Dickinson, Franklin Lakes, NJ), AC133 (Miltenyi Biotech), CD54 (Immunotech, Fullerton, Calif), CD62E (BioSource, Inc, Worcester, Mass), vascular endothelial growth factor receptor-2 (VEGFR-2), Tie-2, von Willebrand factor, endothelial nitric oxide synthase, CXCR1, CXCR2, and CXCR4 (all Santa Cruz Biotech, Santa Cruz, Calif), and analyzed by four-parameter fluorescence using FACScan (Becton Dickinson). Cells positively selected for CD34 expression were also stained with phycoerythrin-conjugated anti-CD117 mAb (Becton Dickinson) and sorted for bright and dim fluorescence using a Facstar Plus (Becton Dickinson) and a phycoerythrin filter.

\section{Indium 111 Labeling of Bone Marrow-derived CD34+ Progenitors}

G-CSF-mobilized cells were immunoselected for CD34+ expression and resuspended in medium containing $20 \mu \mathrm{Ci}$ of ${ }^{111} \mathrm{In}$ 8-oxyquinoline (oxine) per $10^{8}$ cells as described before. ${ }^{16}$ After washing, $2 \times 10^{6111}$ In 8-oxyquinoline (oxine) labeled CD34+ cells were infused intravenously into the nude rats 24 hours after myocardial infarction or into noninfarcted animals. Twenty-four hours later, animals were humanely killed and organs were harvested. Indium 111 counts in each tissue were measured with a gamma spectrometer and calibrated as desintegration per minute per gram tissue using the counter efficiency. Postlabeling viability exceeded $80 \%$ and preliminary experiments demonstrated adequate cell tracking.

\section{Chemotaxis of Human Bone Marrow-derived Hematopoietic Progenitors}

Highly purified CD34 + CD $117+$ cells $(>98 \%$ purity) were plated in 48 -well chemotaxis chambers fitted with membranes $(8-\mu \mathrm{m}$ pores) (Neuro-Probe, Inc, Gaithersburg, Md). After incubation for 2 hours at $37^{\circ} \mathrm{C}$, chambers were inverted and cells were cultured for 3 hours in medium containing eotaxin or SDF-1 at concentrations of $0.2,1.0$, and $5.0 \mu \mathrm{g} / \mathrm{mL}$. Stem cell factor (SCF) was used as negative control in chemotaxis assays at $0.1 \mu \mathrm{g} / \mathrm{mL}$, a biologically active concentration that induced 2-fold proliferation of $\mathrm{CD} 34+\mathrm{CD} 117^{\text {bright }}$ cells after culture for 96 hours. The membranes were fixed with methanol and stained with Leukostat (Fischer Scientific, Pittsburgh, Pa). Chemotaxis was calculated by counting migrating cells in 10 high-power fields.

\section{Animals, Surgical Procedures, and Injection of Human Cells}

Rowett (rnu/rnu) athymic nude rats (Harlan Sprague Dawley, Inc, Indianapolis, Ind) were used in studies approved by the Columbia University Institute for Animal Care and Use Committee. After anesthesia, a left thoracotomy was performed, the pericardium was 
opened, and the left anterior descending (LAD) coronary artery was ligated. Sham-operated rats had a similar surgical procedure without having a suture placed around the coronary artery. For studies on cellular migration, $2.0 \times 10^{6}$ DiI-labeled CD34+ cells obtained from a single donor after G-CSF mobilization were injected into the tail vein 48 hours after LAD ligation in the presence or absence of mAbs with known inhibitory activity against CCR3 $(100 \mu \mathrm{g})$, CXCR4 $(200 \mu \mathrm{g})$, CD34 $(100 \mu \mathrm{g})$, or isotype control antibodies. Inhibiting antibodies were injected in the ischemic area using 3 to 5 injections. Control animals received saline after LAD ligation, and $2.0 \times 10^{6} \mathrm{CD} 34+$ human cells were also injected into the tail vein of sham-operated or LAD-ligated rats receiving three intramyocardial injections of $1.0 \mu \mathrm{g} / \mathrm{mL}$ eotaxin, SDF-1, VEGF, SCF, or saline.

\section{Quantitation of Cellular Migration Into Tissues}

After intravenous injection of human cells, quantitative analysis of the proportion of human cells in rat bone marrow and heart was performed by assessment of both DiI fluorescence and expression of major histocompatibility complex (MHC) class I proteins in rats humanely killed 2 days after injection. Single cell suspension of rat bone marrow was stained with fluorescein-conjugated mAbs against human CD34 and MHC class I beta2 microglobulin (Accurate Chemical \& Scientific Corporation, Meriden, Conn) and analyzed by multiparameter fluorescence using FACScan (Becton Dickinson,), as described previously. ${ }^{17}$ The proportion of human cells in rat heart tissue was expressed as the number of Dil-positive cells per high-power field (minimum 5 fields examined per sample) and as the proportion of cells staining positive for human MHC class I beta2 microglobulin (Accurate Chemical \& Scientific Corporation). Cardiac tissue was stained by immunoperoxidase technique using an Avidin/Biotin Blocking Kit, a rat-absorbed biotinylated antimouse immunoglobulin G, and a peroxidase-conjugate (all Vector Laboratories, Burlingame, Calif). The human origin of the detected cells was confirmed by staining against the human-mitochondrial epitope S-100 (S1-61; Santa Cruz Biotech).

\section{Measurement of Rat Myocardial Chemokine Messenger RNA (mRNA) Expression}

Total RNA was isolated from heart tissues of 3 normal and 12 LADligated rats using the RNAqueous kit from Ambion (Austin, Tex) and was converted to complementary DNA with SuperScript First Strand Synthesis System for RT-PCR from Invitrogen (Carlsbad, Calif). Reverse-transcriptase polymerase chain reaction was used to quantify myocardial expression of rat eotaxin, regulated on activation, normal T expressed and secreted (RANTES), monocyte chemotactic protein-3 (MCP-3), and SDF-1 mRNA at baseline, and at 6, 12, 24, and 48 hours after LAD ligation after normalizing for rat RNA content using rat ribosomal protein L32 (RPL32) mRNA expression. Primer sequences for rat SDF-1 alpha were $5^{\prime}$-CTGTTGTGCTTACTTGTTT AAGGCTTTGTC- $3^{\prime}$ for forward primer and $5^{\prime}$-GACGCCAAGGTC GTCGGT- $3^{\prime}$ for reverse primer. For rat ribosomal protein $\mathrm{L} 32$, primers were $5^{\prime}$-CCCTTCGGCCTCTGGTGAAGC- $3^{\prime}$ for forward primer and 5'-GAACACAAAAACAGGCACACAAGCCATC- $3^{\prime}$ for reverse primer. Primer sequences for rat MCP-3 5'-TTTCACCGTGCACG TGTGGG- $3^{\prime}$ for forward primer and $5^{\prime}$-GTCTTCAGGGCTTTGG AGTTG-3' for reverse primer. Primer sequences for rat RANTES were $5^{\prime}$-ACCTGCCTCCCCATATGGCT-3' for forward primer and $5^{\prime}$-GTATTCTTGAACCCACTTCTTC- $3^{\prime}$ for reverse primer. Primer sequences for rat eotaxin were $5^{\prime}$-TTCTATTCCTGCTGCTCA-3 for forward primer and $5^{\prime}$-CCTGGACCCACTTTTTCT $-3^{\prime}$ for reverse primer. PCR was performed with the GeneAmp PCR System 9700 (ABI, Foster City, Calif). Amplification was for 1 minute at 94 cycles, 27 cycles (RPL32) or 32 cycles (SDF-1, RANTES, MCP-3) with 30 seconds at 94 cycles and 1 minute at 68 cycles, followed by 5 minutes at 68 cycles. PCR products were analyzed on agarose gel stained with ethidium bromide. Reverse-transcriptase polymerase chain reaction products were scanned and quantified by UN-SCAN-IT software from Silk Scientific Inc (Orem, Utah). Values of eotaxin, RANTES, MCP-3, and SDF-1 expression were calculated relative to the values of RPL32 expression. Data were expressed as the means obtained from three independent rats for each time point.

\section{Quantitation of Capillary Density}

To quantitate capillary density and species origin of the capillaries, we stained additional sections with mAbs directed against rat or human CD31 (AbD Serotec, Oxford, United Kingdom, and Research Diagnostics, Inc, Flanders, NJ, respectively), factor VIII (Dako, Carpinteria, Calif), and rat or human MHC class I (Accurate \& Scientific Corporation), as described before. ${ }^{17}$

\section{Measurement of Myocyte Apoptosis by DNA End Labeling of Paraffin Tissue Sections}

For in situ detection of apoptosis at the single cell level, we used the terminal deoxynucleotidyl transferase-mediated dUTP nick end labeling method of DNA end labeling mediated by dexynucleotidyl transferase (Boehringer Mannheim, Mannheim, Germany), as previously described. ${ }^{17}$

\section{Quantification of Infarct Size}

A Masson trichrome stain was performed to evaluate collagen content. This enabled measurement of the size of the myocardial scar using a digital image analyzer. Infarct area was measured with a planimeter digital image analyzer and expressed as a percentage of the total ventricular circumference.

\section{Analyses of Myocardial Function}

Echocardiographic studies were performed with a high-frequency linear array transducer (SONOS 5500, Hewlett Packard, Andover, Mass). Two-dimensional images were obtained at midpapillary and apical levels. End-diastolic (EDV) and end-systolic (ESV) left ventricular volumes were obtained by biplane area-length method, and percent left ventricular ejection fraction was calculated as $([\mathrm{EDV}-\mathrm{ESV}] / \mathrm{EDV}) \times 100$.

\section{F-Fluoro-2-Deoxy-D-Glucose (FDG) Positron Emission Tomography}

Imaging studies were performed in rats using a Concorde $\mathrm{R} 4 \mu \mathrm{PET}$ small animal positron emission tomography imaging system after administration of FDG as described in a previous publication. ${ }^{17}$

\section{Results \\ G-CSF Mobilized Human Bone Marrow Angioblasts Selectively Migrate to Ischemic Myocardium in Vivo In the absence of ischemia, the majority of intravenously in- jected human cells were trapped in the rat liver, spleen, and}




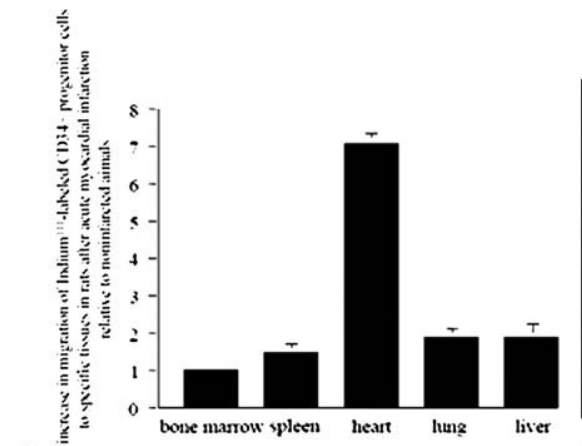

A

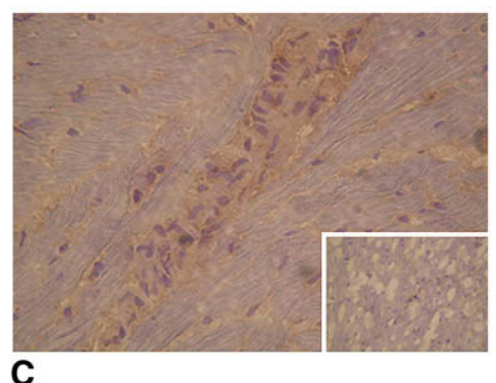

Figure 1. G-CSF mobilized human bone marrow CD34+ prognitors selectively migrate to ischemic myocardium in vivo. A, Detection of indium 111labeled human bone marrow CD34+ progenitors at different organs. B, Fluorescence microscopy shows positive cell engraftment in the infarct area as detected by Dil fluorescence (the side panel represents a negative control). C, Verification of cell engraftment by detection of MHC class I beta-2 microglobulin in the ischemic area by means of immunohistochemistry (the side panel represents a negative control). D, The human origin of the cells was confirmed by staining against the human mitochondrial epitope S-100. lungs, with only $3.2 \%$ migrating to the nonischemic heart. In contrast, after LAD ligation, $23 \%$ of the intravenously injected human cells were present in the heart 24 hours after injection $(P<.01)$. Moreover, the heart was the only rat organ demonstrating a significant increase in migration of human cells after acute myocardial ischemia, with 7.1-fold increase in the indium 111 desintegration per minute count relative to nonischemic myocardium $(P<.01)$ (Figure 1$)$. A detailed examination of cell migration to the heart revealed that $69.1 \%$ and $25.4 \%$ of the cells were detected at the infarction area and the periinfarct zone, respectively. Detection of injected cells at nonischemic myocardium accounted for less than $5 \%$.

\section{G-CSF-Mobilized Human Bone Marrow Angioblasts Express CCR3 and CXCR4 Chemokine Receptors}

To identify potential chemokine receptor-ligand interactions involved in chemotaxis of G-CSF-mobilized angioblasts to the ischemic myocardium, we examined the $\mathrm{CD} 34+\mathrm{CD} 117^{\text {bright }}$ human angioblasts for surface expression of chemokine receptors, specifically the CCR3, which binds the $\mathrm{CC}$ chemokines eotaxin, RANTES, and MCP-3, and the CXCR4, which binds the CXC chemokine SDF-1. Shown in Figure 2, $A$, is CD117 expression on G-CSF-mobilized human CD34+ cells, identifying the dual staining $\mathrm{CD} 117^{\text {bright }}$ population previously shown to contain the angioblast fraction. The CD34+CD117 ${ }^{\text {bright }}$ population expressed both CCR3 and CXCR4 receptors.
Ligation of CCR3 and CXCR4 Chemokine Receptors Induces in Vitro Migration of G-CSF-Mobilized Human Bone Marrow Angioblasts

To examine the functional activity of CCR3 and CXCR4 receptors on the angioblasts, we measured in vitro chemotaxis of CD34+CD117 $7^{\text {bright }}$ human bone marrow cells after triggering these receptors with their specific ligands, respectively, eotaxin and SDF-1. As shown in Figure 2, $B$, human angioblasts demonstrated significant in vitro chemotactic responses to both eotaxin and SDF-1. In contrast, SCF, used as a negative control, was not chemotactic for this cellular population at a biologically active concentration, which induced 2-fold proliferation of $\mathrm{CD} 34+\mathrm{CD} 117^{\text {bright }}$ cells after culture for 96 hours.

Acute Myocardial Ischemia Is Associated With Increased mRNA Expression of CCR3-binding Chemokines, but not CXCR4-binding Chemokines

Next, we examined rat myocardial tissue at various timepoints after LAD ligation to determine whether there was induced mRNA expression of the CCR3-binding CC chemokines eotaxin, RANTES, and MCP-3, and of the CXCR4binding CXC chemokine SDF-1. As shown in Figure 3, A, after LAD ligation, rat myocardium demonstrated a time-dependent increase in eotaxin mRNA expression, with 3-fold induction above baseline being seen at 12 hours, and elevated levels returning to normal by 48 hours $(P<.001)$. Induced expression of RANTES and MCP-3 mRNA was also noted, 
with maximal levels by 12 hours (Figure $3, B$ and $C$; both $P<.01)$. In contrast, by 12 hours after acute myocardial ischemia, SDF-1 mRNA expression in the heart decreased by a mean of $43 \%(P<.01$; Figure $3, D)$.

CCR3-binding Chemokines Regulate Migration of Human CD34 + Progenitors to Ischemic Myocardium, Whereas CXCR4 Binding Regulates Migration to Bone Marrow

We next sought to directly demonstrate that interactions between myocardial CCR3-binding chemokines and CCR3 receptors on human $\mathrm{CD} 34+$ progenitors contributed to myocardial homing of bone marrow angioblasts in LAD-ligated rats. As shown in Figure 4, A, co-administration of a blocking $\mathrm{mAb}$ against CCR3 reduced myocardial trafficking of intravenously injected human bone marrow-derived CD34+ progenitors at 48 hours by a mean of $60 \%$ relative to isotype control antibodies $(P<.01)$. In contrast, mAbs directed to other surface receptors present on these cells, such as CD34 or VEGF-R, as well as against CXCR4, did not inhibit migration to the ischemic myocardium. Moreover, antiCXCR4 mAb inhibited migration of human CD34+ progenitors to rat bone marrow by a mean of $80 \%(P<.01)$, whereas isotype control antibodies were without effect (Figure $4, B$ ).

Manipulating CCR3- and CXCR4-dependent Interactions Increases Migration of Human CD34+ Progenitors to Myocardial Tissue

To definitively prove that CCR3-dependent interactions could redirect human $\mathrm{CD} 34+$ bone marrow progenitors to the heart in vivo, we injected human recombinant eotaxin at $1.0 \mu \mathrm{g} / \mathrm{mL}$ into noninfarcted rat hearts concomitantly with intravenous injection of human angioblasts. As shown in Figure $4, C$, this resulted in 2.3-fold increases in myocardial
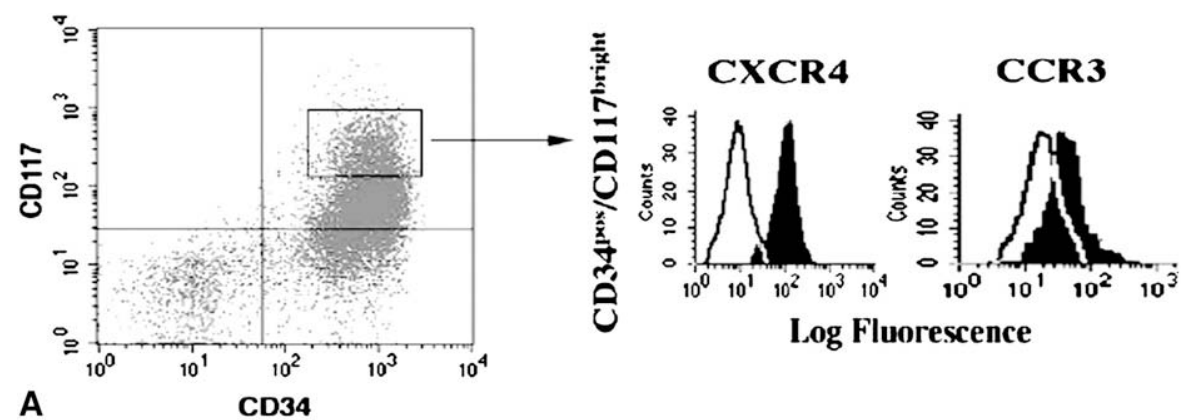

A

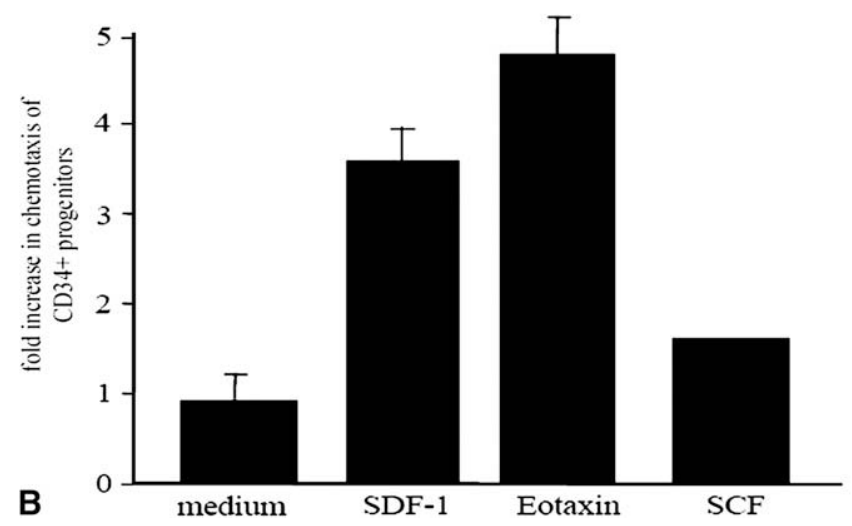

Figure 2. G-CSF mobilized human bone marrow-derived angioblasts express chemokine receptors CCR3 and CXCR4, and demonstrate in vitro chemotactic responses to their ligands, eotaxin and SDF-1. A, Four-parameter flow cytometric phenotypic characterization of $\mathbf{G}$ CSF-mobilized bone marrow-derived cells obtained by leukopharesis from a representative human donor adult. Only live cells were analyzed, as defined by 7-AAD staining. For each marker used, open areas represent background log fluorescence relative to isotype control antibody. The angioblast fraction has previously been characterized to reside in the minor CD34+ population expressing CD117 brightly. The CD34+CD117 ${ }^{\text {bright }}$ angioblast subset expresses CXCR4 and CCR3. The injected CD34+ cells had a purity of greater than $98 \%$; $90 \%$ to $95 \%$ co-expressed the hematopoietic lineage marker CD45, $60 \%$ to $80 \%$ co-expressed the SCF receptor CD117, and less than $1 \%$ co-expressed the monocyte/macrophage lineage marker CD14. B, Results of in vitro chemotaxis of human angioblasts in response to various conditions using a 48-well chemotaxis chamber (Neuro Probe, Inc, Gaithersburg, Md). Chemotaxis is defined as the number of migrating cells per high-power field after examination of 10 high-power fields per condition tested. Chemotaxis is increased in response to eotaxin and SDF-1 (both $P<.01$ ), but not SCF (results are expressed as mean \pm SEM of 3 separate experiments). 


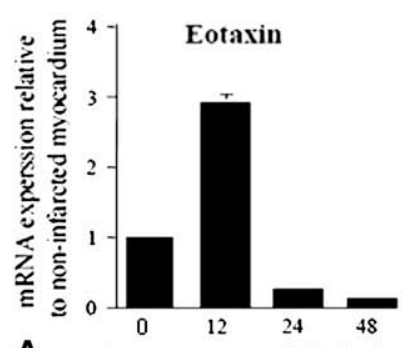

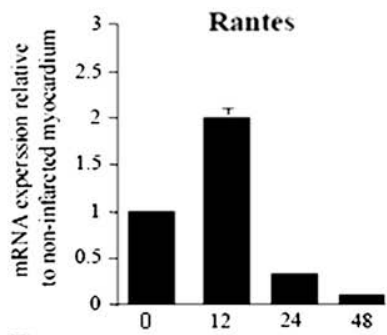

B hours post myocardial infarction

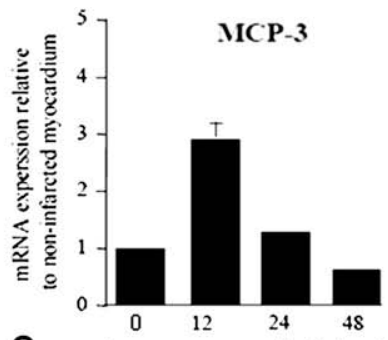

hours post myocardial infarction

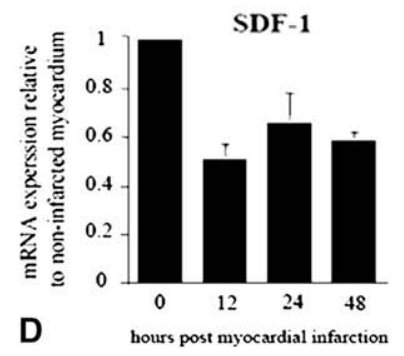

Figure 3. Increased myocardial mRNA expression of CCR3-binding chemokines, but not CXCR4-binding chemokines, after acute ischemia. A to $D$, Chemokine mRNA expression in rat myocardial tissue examined at various time-points after LAD ligation relative to constitutive expression of RPL32 to determine whether there was induced mRNA expression of the CCR3-binding CC chemokines eotaxin, RANTES, and MCP-3, and of the CXCR4-binding CXC chemokine SDF-1. Induced expression of eotaxin, RANTES, and MCP-3 mRNA was noted, with maximal mRNA levels by 6 to 12 hours (all $P<.01$ ). In contrast, by 12 hours after acute myocardial ischemia, SDF-1 mRNA expression decreased by a mean of $43 \%$ in cardiac tissue $(P<.01)$ (results are expressed as mean \pm SEM of 3 separate experiments).

infiltration by human $\mathrm{CD} 34+$ progenitors at 48 hours $(P<$ .01 ), whereas SCF and VEGF, used as negative controls, had little effect. Intramyocardial injection of SDF-1 of $1.0 \mu \mathrm{g} / \mathrm{mL}$ was also effective, inducing a 2.5 -fold increase in angioblast chemotaxis to normal rat hearts $(P<.01)$. However, no increase in myocardial neovascularization was observed at 2 weeks after eotaxin or SDF-1 injection (data not shown), suggesting that additional factors produced under ischemic conditions are necessary for angioblast differentiation into mature hematopoietic cells and subsequent new blood vessel formation.

\section{Intramyocardial SDF-1 Augments}

Neovascularization, Protects Against Cardiomyocyte Apoptosis, and Induces Functional Recovery

We finally examined whether increasing myocardial expression of SDF-1 could result in increased angioblast homing to the ischemic heart and augment angioblast-dependent neovascularization and cardiomyocyte survival after acute ischemia. As shown in Figure 5, $A$ and $B$, co-administration of intramyocardial SDF-1 induced 2-fold greater myocardial neovascularization accompanying intravenous angioblast injection $(P<.01)$ and induced $76 \%$ further reduction in cardiomyocyte apoptosis $(P<.01)$. Moreover, co-administration of intramyocardial SDF-1 together with intravenous angioblasts resulted in an almost 3-fold greater improvement in left ventricular ejection fraction as compared with intravenous injection of angioblasts alone (Figure 5, $C ; P<.01$ ). Quantification of the infarct size showed a $32 \%$ reduction in animals treated with both local SDF-1 application and injection of CD34+ cells, as compared with a $19 \%$ reduction detected after single CD34+ cell injections $(P=.031)$. In addition, myocardial perfusion studies using FDG uptake showed a significantly improved tissue perfusion after combined treatment as compared with CD34+ cell administration alone $(P=.004)$ (Figure 6).

\section{Discussion}

Chemokine receptor-ligand interactions play a major role in leukocyte traffic and recruitment to sites of tissue damage or inflammation. ${ }^{18,19}$ In this study, we have shown that human $\mathrm{CD} 34+$ bone marrow progenitors, which include $\mathrm{CD} 117^{\text {bright }}$ angioblasts or hematopoietic progenitor cells, use two alternative chemokine receptor-ligand pairs to migrate to bone marrow or ischemic myocardium. The site of directed migration was primarily dependent on the specificity of locally induced chemokines, rather than alterations in chemokine receptor expression. Migration to the bone marrow was CXCR4-dependent in response to constitutive expression of the CXCR4-binding chemokine SDF-1 at this site. 

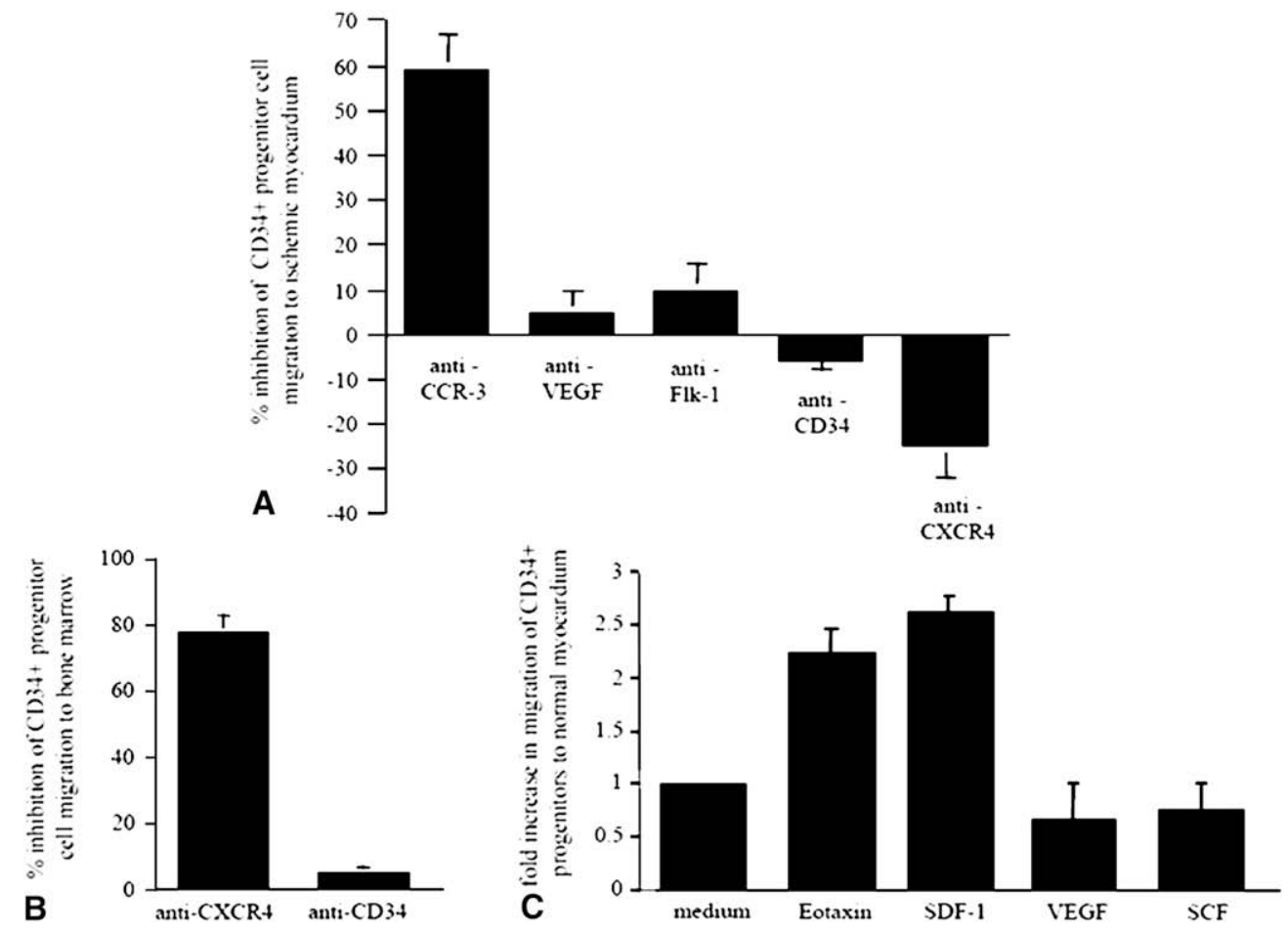

Figure 4. CCR3-binding chemokines regulate migration of human CD34+ progenitors to ischemic myocardium, whereas CXCR4-binding regulates migration to bone marrow. A, Migration of human angioblasts to ischemic rat myocardium at 2 days after intravenous injection is inhibited by antihuman CCR3 mAb $(P<.01)$, but not by mAbs against CXCR4, Flk-1, or isotype control (results are expressed as mean \pm SEM of 3 separate experiments). $B$, the proportion of human $\mathrm{CD} 34+\mathrm{CD}_{117}{ }^{\text {bright }}$ angioblasts in rat bone marrow at 2 days after intravenous injection is significantly decreased by co-administration of anti-CXCR4 mAb (results are expressed as mean \pm SEM of bone marrow studies in 3 animals at each time point). c, Intracardiac injection of eotaxin or SDF-1 at $1 \mu \mathrm{g} / \mathrm{mL}$ significantly increases in vivo chemotaxis of Dil-labeled human angioblasts (98\% CD34 + purity) into nonischemic rat myocardium in comparison with injection of saline or (SCF, both $\boldsymbol{P}<.01$ (results are expressed as mean \pm SEM of 3 separate experiments). Below are shown representative examples of Dil fluorescence microscopy in nonischemic rat hearts after intravenous angioblast administration accompanied by intracardiac injection with eotaxin or SDF-1.

In contrast, migration to ischemic rat myocardium was CCR3- but not CXCR4-dependent, in response to induced mRNA expression of the CCR3-binding chemokines eotaxin, RANTES, and MCP-3, but not SDF-1. However, intramyocardial injection of SDF-1 redirected migration of human angioblasts to ischemic rat hearts, resulting in augmented neovascularizarion, enhanced cardiomyocyte survival, and functional cardiac recovery. These results demonstrate the feasibility of manipulating chemokine receptor-ligand interactions for therapy of cardiovascular disease and suggest that augmenting CXCR4-dependent interactions at extrahematopoietic sites could provide a general paradigm for enhancing hypoxic or ischemic tissue repair by bone marrow-derived hematopoietic progenitors. Our study elaborated on the role of CCR3- and CXCR4-mediated chemoattraction in the early phase after acute myocardial infarction, and data on the midterm and long-term cell survival in ischemic myocardium were not included.
Homing and retention of hematopoietic progenitors in mammalian bone marrow are processes controlled by a number of adhesive interactions between stromal cells and hematopoietic progenitors. ${ }^{2,3}$ Binding of SDF-1 to its ligand, CXCR4, results in augmented interactions between integrin receptor-ligand pairs, including very late antigen-4 and vascular cell adhesion molecule (VCAM-1)/CD106, ${ }^{19}$ which serve to tether and retain very late antigen-4 expressing progenitors to VCAM-1/CD106 positive stromal cells in the bone marrow. Systemic treatment with G-CSF induces activation of neutrophil precursors in the bone marrow and their subsequent secretion of neutrophil proteases, which directly cleave the $N$-terminal regions of SDF-1 and CXCR4, as well as VCAM-1 ${ }^{6,7}$ Inasmuch as this results in egress of hematopoietic progenitors from the bone marrow into the peripheral circulation, G-CSF administration may facilitate myocardial migration of $\mathrm{CD} 34+\mathrm{CD} 117^{\text {bright }}$ angioblasts in response to CCR3-binding chemokines induced in the ischemic heart. 


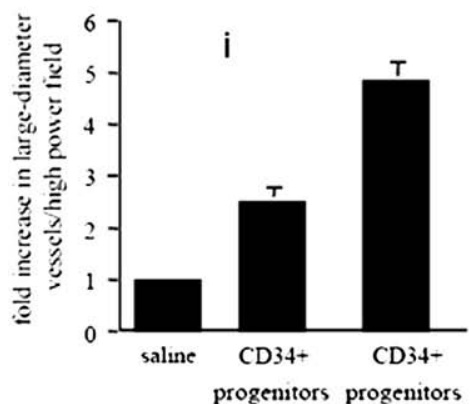

A

SDF-1
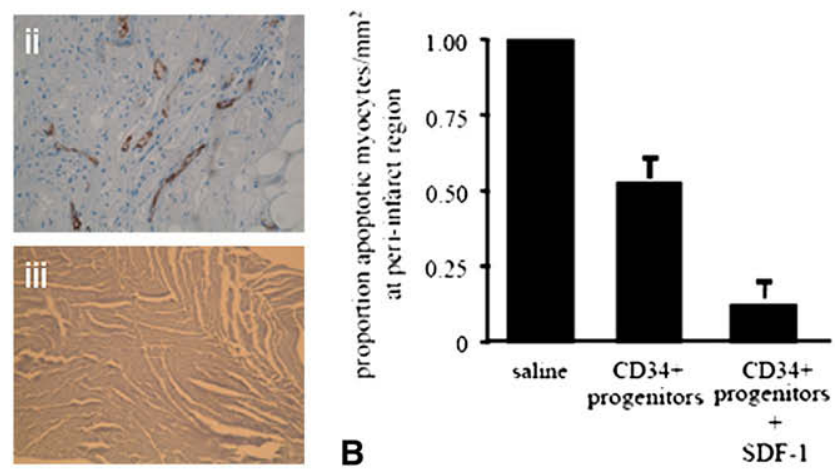
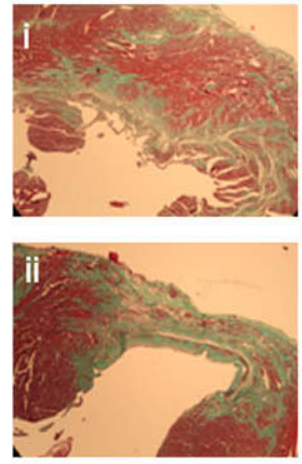

C

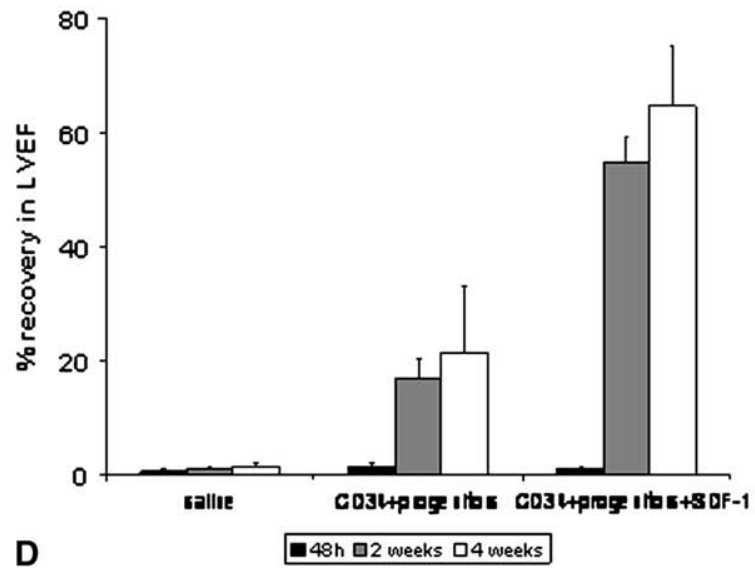

Figure 5. Intramyocardial injection of SDF-1 increases angioblast chemotaxis to ischemic myocardium, augmenting neovascularization, cardiomyocyte survival, and functional cardiac recovery. Intracardiac injection of SDF-1 into infarcted rat hearts in combination with intravenously-injected CD34 + human bone marrow cells resulted in a further 2-fold increase in capillary numbers (A), in a further reduction in cardiomyocyte apoptosis of $76 \%$ (B), and in a further reduction in infarct size (C) as compared with intravenously-injected angioblasts alone (all $P<.001$ ). The results of vascular density are expressed as mean \pm SEM of 3 separate experiments. Large diameter vessels include vessels built by more than 6 nuclei and with a diameter greater than $20 \mu \mathrm{m}$. Representative staining against factor VIII and Masson trichrome stain shows increased vasculary density and reduced infarct scar, respectively, in treated animals (i) as compared with controls (ii). (D). Intracardiac co-administration of SDF-1 results in 4-fold greater improvement in left ventricular ejection fraction, determined by echocardiography, compared with intravenous injection of CD34 + human bone marrow cells alone $(P<.01)$. Results are expressed as mean \pm SEM of 3 separate experiments.

Eotaxin is principally produced by smooth muscle cells and fibroblasts in subendothelial tissue locations, ${ }^{20}$ and its expression is typically associated with the recruitment of eosinophils and basophils to inflamed tissues and their accumulation during certain inflammatory processes, such as allergy and asthma. ${ }^{21}$ The eotaxin receptor, CCR3, is expressed on eosinophils, basophils, mast cells, and the Th2 subset of T cells, and also binds the chemokines RANTES and MCP-3 with high affinity, but not other CC or CXC chemokines. ${ }^{22-24}$ Increased levels of eotaxin in autoimmune myocardial inflammation and during rejection episodes of transplanted heart confirm its crucial role as chemoattractant in myocardial tissue..$^{25,26}$

Constitutive expression of CCR3 has also been reported by human and mouse bone marrow progenitors as well as dendritic cells. ${ }^{14,15}$ However, whereas CD34+ eosinophil and dendritic bone marrow-derived progenitors demonstrate CCR3-dependent chemotactic responses, ${ }^{23-25}$ mouse hematopoietic stem cells that home to the bone marrow and recapitulate hematopoiesis do not. ${ }^{13}$ These observations suggest that CCR3-dependent chemokine interactions direct migration of bone marrow-derived leukocyte progenitors away from the marrow to distal sites of tissue damage and inflammation. In support of these conclusions, CCR3-deficient mice demonstrate altered migration of eosinophils and mast cells, respectively, to the intestine and lungs. ${ }^{24}$ Our results extend these observations to migration of human $\mathrm{CD} 34+\mathrm{CD} 117^{\text {bright }}$ bone marrow progenitors to the site of myocardial ischemia where expression of eotaxin, RANTES, and MCP-3 mRNA 


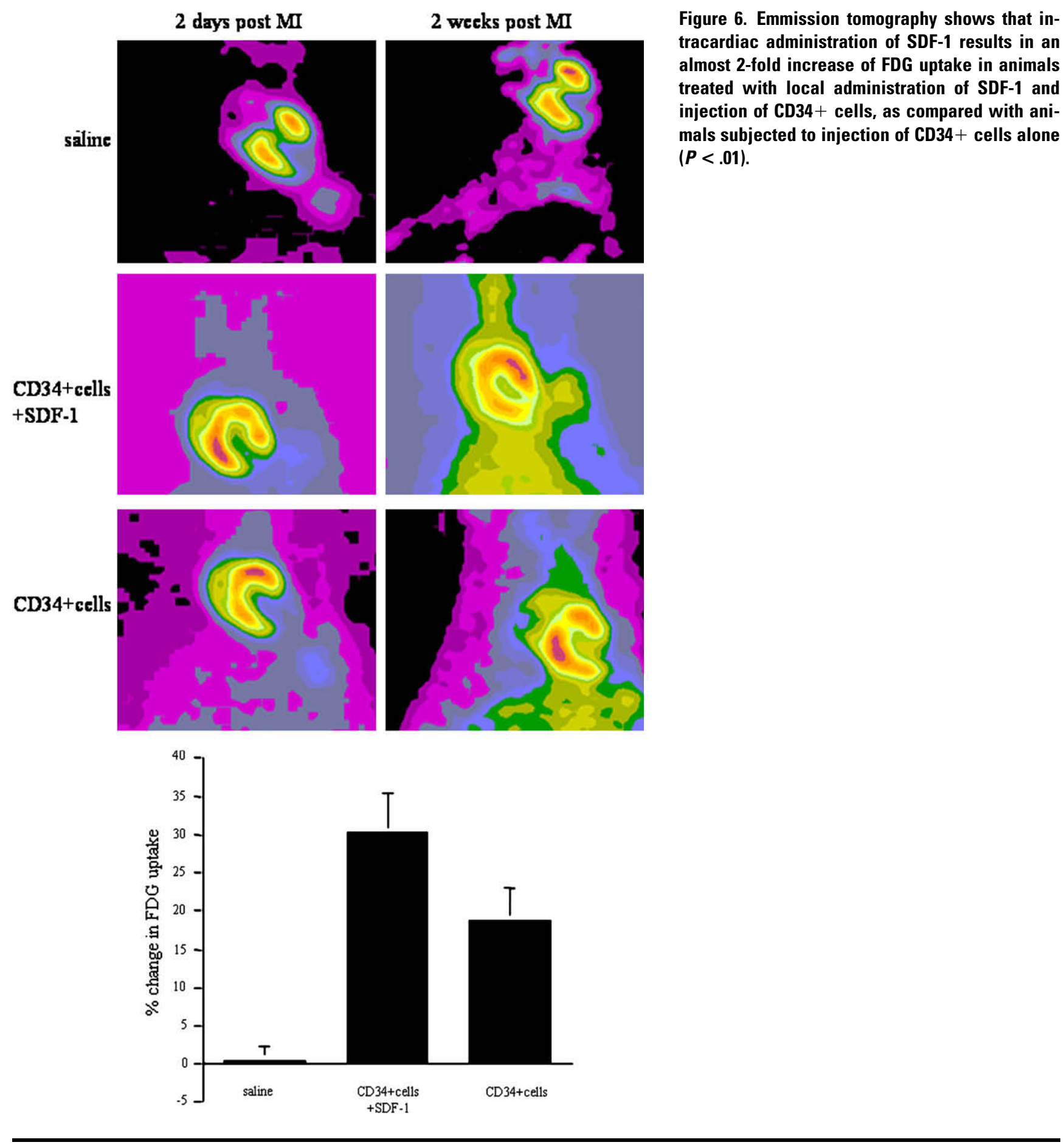

was induced. Moreover, since hypoxia is a stimulus for induction of eotaxin mRNA, ${ }^{27}$ we hypothesize that CCR3-directed chemotaxis of human CD34+CD117 $7^{\text {bright }}$ angioblasts or hematopoietic progenitors contributes to neovascularization of hypoxic or damaged tissues throughout the body.

SDF-1, a biologically active chemotactic factor for human endothelial progenitors, ${ }^{28}$ augmented angioblast-dependent myocardial chemotaxis, neovascularization, cardiomyocyte survival, and functional cardiac recovery when directly injected into the ischemic myocardium. Together with results from adult humans in whom SDF-1 expression at extrahematopoietic sites is accompanied by aberrant neovascularization, ${ }^{28}$ our data demonstrate that SDF-1 expression at sites outside the bone marrow can play a major role in induction 
of tissue neovascularization by bone marrow-derived hematopoietic progenitors. These conclusions are supported by recent studies using SDF-1 in an animal model of reduced hind limb perfusion ${ }^{29}$ and in genetically engineered skeletal myoblasts implanted into ischemic myocardium. ${ }^{30}$

Together, our results suggest that redirected migration of CD34+ progenitors from the bone marrow to sites of acute injury may represent a general mechanism by which endogenous repair of damaged tissues occurs, and that the specificity of the migratory pattern is governed by the induced chemokine profile in a given injured tissue. Moreover, our results suggest that it may be possible to selectively activate CXCR4- or CCR3-dependent chemotactic pathways to direct migration of $\mathrm{CD} 34+$ progenitors to sites of tissue ischemia or damage and induce therapeutic neovascularization for tissue repair. The effect of this combined approach using autologous cells injections in immunocompetent hosts is required to address the question of clinical applicability.

\section{References}

1. Wright DE, Wagers AJ, Gulati AP, Johnson FL, Weissman IL. Physiological migration of hematopoietic stem and progenitor cells. Science. 2001;294:1933-6.

2. Frenette PS, Subbarao S, Mazo IB, von Andrian UH, Wagner DD. Endothelial selectins and vascular cell adhesion molecule-1 promote hematopoietic progenitor homing to bone marrow. Proc Natl Acad Sci U S A. 1998;95:14423-8

3. Verfaillie CM. Adhesion receptors as regulators of the hematopoietic process. Blood. 1998;92:2609-12.

4. Mohle R, Bautz F, Rafii S, Moore MA, Brugger W, Kanz L. The chemokine receptor CXCR-4 is expressed on CD34+ hematopoietic progenitors and leukemic cells and mediates transendothelial migration induced by stromal cell-derived factor-1. Blood. 1998;91:4523-30.

5. Honczarenko M, Le Y, Swierkowski M, Ghiran I, Glodek AM, Silberstein LE. Human bone marrow stromal cells express a distinct set of biologically functional chemokine receptors. Stem Cells. 2006; 24:1030-41.

6. Lévesque JP, Hendy J, Takamatsu Y, Simmons P, Bendall LJ. Disruption of the CXCR4/CXCL12 chemotactic interaction during hematopoietic stem cell mobilization induced by GCSF or cyclophosphamide. J Clin Invest. 2003;111:187-96.

7. Petit I, Szyper-Kravitz M, Nagler A, Lahav M, Peled A, Habler L, et al. G-CSF induces stem cell mobilization by decreasing bone marrow SDF1 and up-regulating CXCR4. Nat Immunol. 2002;3:687-94.

8. Lagasse E, Connors H, Al-Dhalimy M, Reitsma M, Dohse M, Osborne L, et al. Purified hematopoietic stem cells can differentiate into hepatocytes in vivo. Nat Med. 2000;6:1229-34.

9. Krause DS, Theise ND, Collector MI, Henegariu O, Hwang S, Gardner R, et al. Multi-organ, multi-lineage engraftment by a single bone marrow-derived stem cell. Cell. 2001;105:369-77.

10. Kocher AA, Schuster MD, Szabolcs MJ, Takuma S, Burkhoff D, Wang J, et al. Neovascularization of ischemic myocardium by human bone-marrow-derived angioblasts prevents cardiomyocyte apoptosis, reduces remodeling and improves cardiac function. Nat Med. 2001;7:430-6.

11. Schächinger V, Assmus B, Britten MB, Honold J, Lehmann R, Teupe C, et al. Transplantation of progenitor cells and regeneration enhancement in acute myocardial infarction: final one-year results of the TOPCAREAMI Trial. J Am Coll Cardiol. 2004;44:1690-9.

12. Stamm C, Westphal B, Kleine HD, Petzsch M, Kittner C, Klinge H, et al. Autologous bone-marrow stem-cell transplantation for myocardial regeneration. Lancet. 2003;361:45-6.
13. Wright DE, Bowman EP, Wagers AJ, Butcher EC, Weissman IL. Hematopoietic stem cells are uniquely selective in their migratory response to chemokines. J Exp Med. 2002;195:1145-54.

14. Lamkhioued B, Abdelilah SG, Hamid Q, Mansour N, Delespesse G, Renzi PM. The CCR3 receptor is involved in eosinophil differentiation and is up-regulated by $\mathrm{Th} 2$ cytokines in CD34+ progenitor cells. $J$ Immunol. 2003;170:537-47.

15. Lataillade JJ, Clay D, Dupuy C, Rigal S, Jasmin C, Bourin P, et al. Chemokine SDF-1 enhances circulating CD34(+) cell proliferation in synergy with cytokines: possible role in progenitor survival. Blood. 2000;95:756-68.

16. Chin BB, Nakamoto Y, Bulte JW, Pittenger MF, Wahl R, Kraitchman DL. 111 In oxine labelled mesenchymal stem cell SPECT after intravenous administration in myocardial infarction. $\mathrm{Nucl} \mathrm{Med}$ Commun. 2003;24:1149-54.

17. Kocher AA, Schuster MD, Bonaros N, Lietz K, Xiang G, Martens TP, et al. Myocardial homing and neovascularization by human bone marrow angioblasts is regulated by IL-8/Gro CXC chemokines. $J$ Mol Cell Cardiol. 2006;40:455-64.

18. Vandervelde S, van Luyn MJ, Tio RA, Harmsen MC. Signaling factors in stem cell-mediated repair of infarcted myocardium. J Mol Cell Cardiol. 2005;39:363-76.

19. Shen W, Bendall LJ, Gottlieb DJ, Bradstock KF. The chemokine receptor CXCR4 enhances integrin-mediated in vitro adhesion and facilitates engraftment of leukemic precursor-B cells in the bone marrow. Exp Hematol. 2001;29:1439-47.

20. Teran LM, Mochizuki M, Bartels J, Valencia EL, Nakajima T, Hirai K, et al. Th1- and Th2-type cytokines regulate the expression and production of eotaxin and RANTES by human lung fibroblasts. Am J Respir Cell Mol Biol. 1999;20:777-86.

21. Garcia-Zepeda EA, Rothenberg ME, Ownbey RT, Celestin J, Leder P, Luster AD. Human eotaxin is a specific chemoattractant for eosinophil cells and provides a new mechanism to explain tissue eosinophilia. Nat Med. 1996;2:449-56.

22. Daugherty BL, Siciliano SJ, DeMartino JA, Malkowitz L, Sirotina A, Springer MS. Cloning, expression, and characterization of the human eosinophil eotaxin receptor. J Exp Med. 1996;183:2349-54.

23. Sallusto F, Mackay CR, Lanzavecchia A. Selective expression of the eotaxin receptor CCR3 by human T helper 2 cells. Science. 1997;277: 2005-7.

24. Humbles AA, Lu B, Friend DS, Okinaga S, Lora J, Al-Garawi A, et al. The murine CCR3 receptor regulates both the role of eosinophils and mast cells in allergen-induced airway inflammation and hyperresponsiveness. Proc Natl Acad Sci U S A. 2002;99:1479-84.

25. Göser S, Andrassy M, Buss SJ, Leuschner F, Volz CH, Ottl R, et al. Cardiac troponin I but not cardiac troponin $\mathrm{T}$ induces severe autoimmune inflammation in the myocardium. Circulation. 2006;114: 1693-702.

26. Melter M, Exeni A, Reinders ME, Fang JC, McMahon G, Ganz P, et al. Expression of the chemokine receptor CXCR3 and its ligand IP-10 during human cardiac allograft rejection. Circulation. 2001; 104:2558-64

27. Moayeri M, Haines D, Young HA, Leppla SH. Bacillus anthracis lethal toxin induces TNF-alpha-independent hypoxia-mediated toxicity in mice. J Clin Invest. 2003;112:670-82.

28. Moore MA, Hattori K, Heissig B, Shieh JH, Dias S, Crystal RG, et al. Mobilization of endothelial and hematopoietic stem and progenitor cells by adenovector-mediated elevation of serum levels of SDF-1, VEGF, and angiopoietin-1. Ann N Y Acad Sci. 2001;938: 36-45.

29. Yamaguchi J, Kusano KF, Masuo O, Kawamoto A, Silver M, Murasawa S, et al. Stromal cell-derived factor-1 effects on ex vivo expanded endothelial progenitor cell recruitment for ischemic neovascularization. Circulation. 2003;107:1322-8.

30. Askari AT, Unzek S, Popovic ZB, Goldman CK, Forudi F, Kiedrowski M, et al. Effect of stromal-cell-derived factor 1 on stemcell homing and tissue regeneration in ischaemic cardiomyopathy. Lancet. 2003;362:697-703. 